\title{
Human Factors/Ergonomics (HFE) in Leadership and Management: Organizational Interventions to Reduce Stress in Healthcare Delivery
}

\author{
Michael R. Privitera \\ Medical Faculty and Clinician Wellness Program, University of Rochester Medical Center, Rochester, NY, USA \\ Email: Michael_Privitera@urmc.rochester.edu
}

How to cite this paper: Privitera, M.R. (2020) Human Factors/Ergonomics (HFE) in Leadership and Management: Organizational Interventions to Reduce Stress in Healthcare Delivery. Health, 12, 1262-1278. https://doi.org/10.4236/health.2020.129091

Received: August 15, 2020

Accepted: September 26, 2020

Published: September 29, 2020

Copyright $\odot 2020$ by author(s) and Scientific Research Publishing Inc. This work is licensed under the Creative Commons Attribution International License (CC BY 4.0).

http://creativecommons.org/licenses/by/4.0/ (c) (i) Open Access

\begin{abstract}
Clinician Burnout is a personal and public health issue. Most occupational stressors contributing to clinician burnout are systemic. The combination of organizational interventions along with individual interventions is necessary to make significant lasting difference in reducing burnout, improving clinician and patient satisfaction and reducing latent error in healthcare delivery. Application of Human Factors/Ergonomics (HFE) science in healthcare leadership and management is a gap in current training for leaders. HFE uses concepts from organizational, educational and cognitive science, systems science and industrial engineering. HFE application is especially necessary in a fast changing highly stressful healthcare environment which impacts the wellbeing of clinicians and the safety of patients under care. Practical suggestions for working with various healthcare leadership styles and organizational dynamics, while aligning wellness efforts with institutional mission are discussed. Concrete examples of decreasing extraneous mental load on clinicians to preserve their brainpower to achieve quality patient care are illustrated. Organizational interventions in combination with individual interventions to reduce and manage burnout have enormous potential to improve clinician wellbeing and satisfaction in taking care of patients, reduce costs, risk of error and create the safe working environment needed to sustainably give high quality care to patients.
\end{abstract}

\section{Keywords}

Leadership, Management, Burnout, Human Factors, Ergonomics, Occupational Stress, Organizational Interventions 


\section{Background}

\subsection{Introduction}

Clinician Burnout has existed in healthcare for decades, but causes, consequences and potential solutions are now better understood [1]. Interventions were individually focused upon addressing stress in medicine using mindfulness, gratefulness, guided imagery and other ways of coping. However, a more current approach recommends addition of organizational interventions to reduce burnout more effectively and sustainably. Burnout has been correlated with increasing health risk to the individual clinician [1]. The greater the severity of burnout, the greater the chance of causing Major Depression [2] heart disease, substance abuse, and suicide [3] [4] [5]. Patient safety and quality of care are affected by clinician burnout as are increased expenses to the institution [6] [7] [8] [9]. The chance of causing a medical error increases and quality of care goes down; patient's experience of receiving care goes down. Tragically, clinicians often do not get help due to fear of real or imagined repercussions on their career that took years of training and sacrifice to achieve [10].

Eighty percent of the occupational stressors creating clinician burnout are organizational [11]. National Academy of Medicine determined twelve major drivers that are influencing burnout in the United States: The healthcare industry, laws regulations and standards, healthcare reform and payment policies, medical record documentation and coding requirements, quality measurement and reporting, prescription drug monitoring programs, maintaining privacy and security, prior authorization processes, professional licensure, maintenance of certification, professional liability and the patient-physician relationship [12].

In reviews and meta-analysis of interventions to reduce burnout in physicians, organizational interventions had larger effects than individually directed interventions [13] [14] [15]. Healthy clinician administrator relationships can create the culture for clinicians to acknowledge and reduce stress [16]. Organizational plus individual intervention pairing may be the optimal framework for mitigating burnout.

In published organizational interventions to reduce burnout, there are substantial variations in approaches, intervention ingredients and intensity. Most controlled studies or single arm pre/post evaluations were ways of reducing workload, providing protected time for peers to meet or work schedule changes. In complex healthcare systems, it is difficult to achieve a high quality controlled study unless variables are simplified and measurable [13] [14] [15]. It may take many years to research a wide variety of interventions in such complex systems. For the average healthcare system struggling today, this time lag is not feasible for those currently feeling the impact of burnout. See Table 1 for a summary of some key publications on organizational interventions to reduce burnout in healthcare.

This paper will augment other organizational intervention literature by offering a theory-based approach of Human Factors/Ergonomics to reduce occupational 
Table 1. Selected references: Individual and systemic/organizational interventions to reduce healthcare worker burnout.

\section{Reference}

West, CP, Dyrbye LN et al. Interventions to prevent and reduce physician burnout: A systemic review and meta-analysis. The Lancet. September 28, 2016.

\section{Area(s) of burnout focus Key findings}

Physicians, individual and Individual and organizational strategies can result interventions in clinically meaningful reduction of burnout in physicians. Further research suggested as to how organizational to combine interventions and what most effective in certain populations.

Organization-directed interventions associated with higher treatment effects than individually directed interventions. However, acknowledge diverse interventions and settings.

Meta-analysis. JAMA Intern Med. December 5, 2016 E 1-E 11. organizational interventions

Physicians

DeChant PF, Acs A et al. Effect of Organization-Directed Workplace Interventions on Physician Burnout: A Systematic Review. Mayo Clinic. Proc. December 2019 3(4): 384-408.

organizational interventions

Physicians. Leadership led organizational strategies

Shanafelt TD, Noseworthy JH. Executive Leadership and Physician Wellbeing; Nine Organizational Strategies to Promote Engagement and Reduce Burnout. Mayo Clin Proc. January 2017; 92(1): 129-146.

Carayon P, Cassel C, Dzau, VJ. Improving the System to Support All clinicians. System Clinician Well-being and Provide Better Patient Care. JAMA improvements

December 10, 2019. Vol. 322 (22): 2165-2166.

Nowrouzi B, Lightfoot $\mathrm{N}$ et al. Occupational stress management Nurses. Quality of Work and Burnout. Interventions in Nursing and their implications for Life (QWL)

healthy work environments. Workplace Health and Safety. July Workplace interventions 2015 63(7): 308-315.

Shanafelt TD, Ripp J, et al. Caring for healthcare workers during crisis. Creating a resilient organization. American Medical Association. 5 May 8, 2020.

https://www.ama-assn.org/practice-management/sustainability/c reating-resilient-organization-health-care-workers-during

National Academy of Medicine Action Coalition on Clinician Wellbeing. https://nam.edu/clinicianwellbeing/wp-content/uploads/2019/07 well-being and resilience. /Factors-Affecting-Clinician-Well-Being-and-Resilience-July-201 Service vs. personal life 9.pdf

National Academy of Medicine. Taking Action Against Clinician The changing landscape of Burnout: A Systems Approach to Professional Wellbeing. https://nam.edu/systems-approaches-to-improve-patient-care-by System has had profound -supporting-clinician-well-being/ Factors affecting clinician the U.S. Health Care

All healthcare workers. Organizational steps before during and after crisis to coping effects on clinical practice and the experiences of clinicians, students and trainees ("learners"), and patients and their families
Organizational interventions targeted to improve process, team-based care, use of scribes/medical assistants to complete documentation and tasks had largest benefits.

Nine organizational strategies summarized and how to operationalize individually, at work unit, at organizational and addressing issues nationally.

Create positive work environments, learning environments, reduce administrative burden, enable technological solutions, support to clinicians and students, and invest in research.

Person-directed and organization-directed prevention measures expected to prevent burnout and improve workers' mental health.

Pre-existing institutional supports that should be in place, being aware of stresses, the stress injury, stress first aid of basic needs, psychosocial and mental health support, and enhance communications. Organizational assisting in recovery aids to support copping and recovery.

Rules and regulations, society and culture, Personal factors, skills and abilities, healthcare responsibilities, learning/practice environment, organizational factors.

Aligning organizational structures, and processes with workforce values, use systems approach, engage and commit leadership at all levels. Major Goals: Creating positive work and learning environments, reducing administrative burden, enabling technology solutions, providing support, reducing stigma and investing in research.

stress and burnout that has been applied in other industries.

The interventions in this manuscript are based upon what we currently know about the occupational stressors that cause burnout, addressing them, preventing them from happening, or minimizing their impact. An adaptation of the science 
of Human Factor/Ergonomics (HFE) applicable for leaders will be discussed [17]. HFE uses concepts from organizational, educational and clinical psychology, physiology, systems science, cognitive neuroscience and industrial engineering [18]. The goals of HFE are reducing error, improve system performance while creating a safe work environment for the wellbeing of the worker [19]. Patient safety is one component of system performance. HFE helps us understand the mechanisms of impact of high or chronic occupational stress, and then gives us the tools to apply to improve conditions.

Creation of organizational processes to continually improve the work and healing environment, and improvement of communications between rank and file clinicians, leaders and managers will be at the core of suggestions for organizational improvement.

\subsection{Assessing the Organization}

Both individuals and organizations can have coping systems that are dysfunctional.

Questions to consider in assessing the organization: What is the surrounding culture among clinicians and in relationship with their leaders? What is the level of leadership insight, political environment, hierarchy structure, how decisions are made and the role of gatekeepers? What are administrators' goals as you consider your own? What are the leader personalities and rituals of communication? What is the mission of the institution and how can your wellness efforts help the mission [20]? Are leaders conventional thinkers or integrative thinkers?

Conventional thinking focuses only on obviously relevant features, considers one way linear relationships between variables and makes "either-or choices" settling for best available options. Integrative thinking seeks less obvious but relevant factors, considers nonlinear and multidirectional relationships among variables, and examines how parts fit together in the ecosystem and how decisions affect one another. Integrative thinkers creatively resolve tensions between opposing ideas, and generate innovative solutions that are superior to each option alone. Leaders who use integrative thinking are more successful in general than those using conventional thinking [21].

\section{Organizational System Challenges}

\subsection{Opened and Closed Organizational Systems}

General Systems Theory described by Bertalanffy, described "open" and "closed" systems [22]. The system's boundary is the perimeter that encloses the system separating it from its environment, distinguishing system members from nonmembers [20]. In closed hierarchical systems there are also internal boundaries between leadership members and non-members. Boundary permeability reflects the degree of resistance that occurs when trying to move people, ideas and resources into and out of the system or between non-leaders and leaders. The degree of boundary permeability distinguishes open and closed systems. Gatekee- 
pers are those people who control when, where and under what circumstances boundary transactions can occur (See Figure 1).

Academic Medical Centers are an example of closed organizational systems [20]. They demonstrate hierarchy, with strict communication rituals and close attention to rules of communications, adherence to chain of command, rigid definition of values and behavioral etiquette. Mid-level leaders in meetings with senior leadership may, without any malice whatsoever, filter out information that ascends to leadership that misses rank and file requests. Alternatively, less fidelity to the concerns of rank and file may be motivated by fear of the senior leader's response as to how this may affect the security of their own job. Mid-level leaders have latitude to decide upon what information should go to senior leader as part of their job to "protect" the senior leader as well.

Closed systems, at their extreme, demand excessive time and emotional commitment to the organization, as well as extreme demands for loyalty. Management style includes control by fear, force, or persuasion through charisma and authoritarian leadership.

Open organizational family systems have high boundary permeability, little to no gatekeeping, minimal definition of values and behavioral etiquette. Open systems at their extreme tend to exhibit less clear sense of mission or goals binding members together, lower organizational identity and less involved leadership. An example can be found in an aging federal state or county civil service agencies [20].

Organizations can exist on a continuum. The individual member's and system's health is affected by the organization's ability to flexibly move up and down

\section{Hospital Hierarchy Levels and Strength of Gatekeeping of Access to C-Suite}

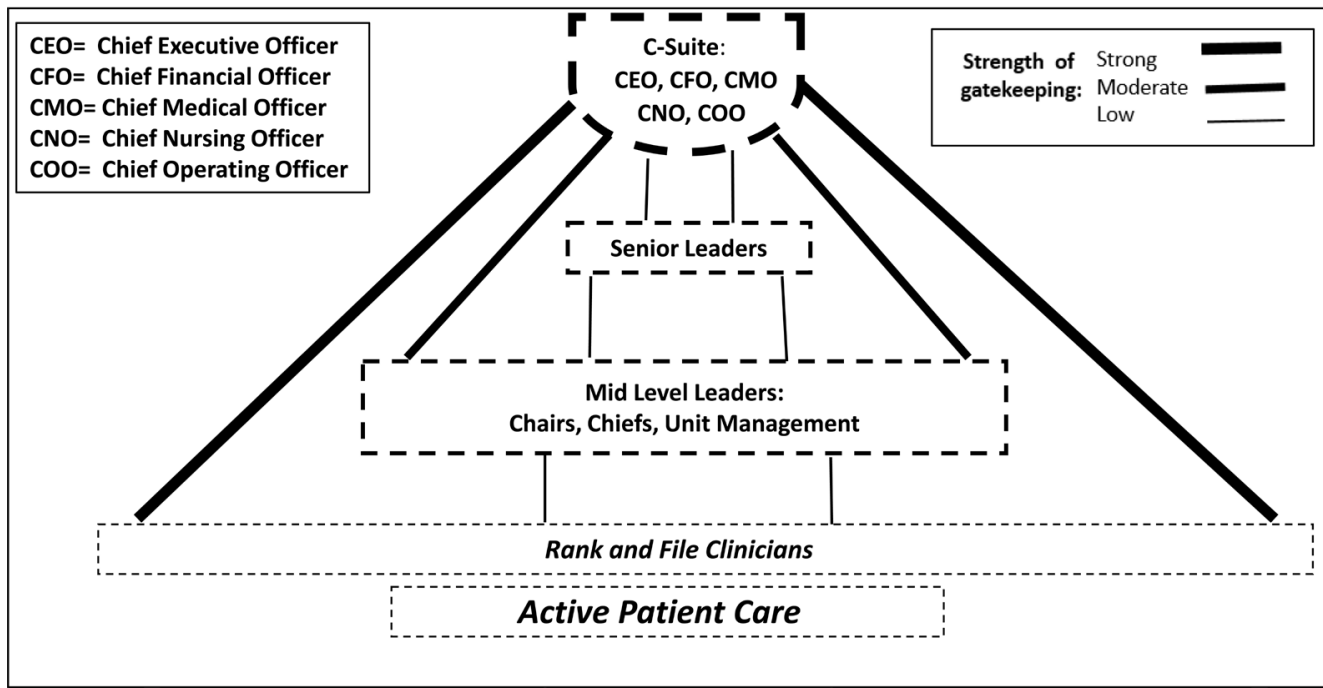

Figure 1. Illustrates gatekeeping processes in closed hierarchical systems. Rank and file clinicians have many levels of hierarchy and custom that prevent direct access to senior leaders. Though processes are reliable and formal, the ability of a closed system to respond rapidly to external environment changes is a disadvantage, and misses the nuances of solutions known to front-line clinicians that could expedite effective improvements at the point of active patient care. 
the continuum between open and closed systems when needed. Occupational distress in the organization can be a symptom of system dysfunction [20].

\subsection{Organization Type and Context to Its External Environment}

In a closed system, the C Suite leadership (Chief Executive Officer, Chief Financial Officer, Chief Medical Officer, etc.) must follow guidance from outside authorities to achieve the financial survival of the organization. Examples include healthcare delivery frameworks that affect revenue to the hospital such as quality and safety of care metrics, patient satisfaction scores, while controlling costs-“Triple Aim" framework [23]. When a C-suite is insulated from real time front line clinicians concerns, there is risk of C-Suite blindness to factors that may not immediately affect finances, but will certainly later on-such as the experiences and human factors involved in the delivery of healthcare [11] [24]. The importance of a national framework of healthcare delivery that includes the fourth aim of human factors in the experience of providing care is underappreciated, and is particularly deleterious to clinicians in closed systems. Without the fourth aim awareness in the C-Suite, unintended negative effects occur, contributing to, aggravating and perpetuating clinician burnout. The national adoption of the "Quadruple Aim" (costs, quality, patient experience and experience of providing care) [24] [25] [26] is critical for sustainable and safe healthcare delivery.

As noted by Institute of Medicine, the most frequent source of errors are due to systemic issues creating latent conditions from decisions made higher up in systems of care [27]. The upstream decisions made by leaders can contribute to both medical error at the point of care and burnout in the clinicians providing the care. This is especially perpetuated in systems having just one way communication-such as leadership to rank and file clinicians, without any mechanisms for leaders to receive feedback about problems occurring at the point of delivering care.

Both in Europe and the United States, regulations, healthcare initiatives, policies and mandatories are mainly developed by management boards, or policymaking bodies with very little input from frontline staff or patients, perpetuating high levels of burnout by this behavior [28].

\subsection{Hospital Leadership Characteristics That Affect Ability to Initiate Clinician Wellbeing Programs}

Consider how an approach to start a clinician wellness initiative may be different in working with each leader characteristics below.

1) Intrinsically motivated to improve clinician wellbeing.

2) Extrinsically motivated to improve clinician wellbeing.

3) Does not understand what to do. Not want to open "Pandora's Box".

4) Not aware there is a problem. Leader is just doing what the authoritative agencies tell them to do.

5) Not interested in clinician wellbeing, profit is the focus.

6) Not too interested, worried what their boss may think, not feel secure in own position, risk-aversive to own career. 
a) Intrinsically motivated to improve clinician wellbeing. This leader tends to think in integrated fashion (between two choices is able to include benefits of both and come up with choice that is superior to both). They tend to know how important the wellbeing of clinicians is to the patients they serve and multiple other hospital impacts. Not many headwinds, and with this support, significant efficiencies occur to push the initiative forward.

b) Extrinsically motivated to improve clinician wellbeing. May be due to national mandates, for example the Accreditation Council for Graduate Medical Education (ACGME) the Clinical Learning Environment Review (CLER) program. CLER requires methods available to measure burnout and provide the information to the resident as well. If this is not followed, accreditation can be terminated. If the senior leaders are not strongly intrinsically motivated, other leaders more enthusiastic at the institution may help with the pace of getting these changes made.

c) Does not understand what to do. Not want to open "Pandora's Box". This leader may ignore the problem until there was no choice but to address it. They may not understand what they need to do, or don't want to be seen as incompetent. It may be helpful to start with education on burnout impact, including leaders and rank and file clinicians at the same time and describe some organizational interventions that may help. Small unit pilot successes may lead the way to understanding there are effective ways to deal with the issues at the institution.

d) Not aware there is a problem. Leader is just doing what agencies are telling them to do. This situation would need opportunity to illustrate why too many mandates, laws, policies, regulations coming from multiple authorities create expectations that are beyond human ability to do and actually backfire in patient safety. This will initially seem paradoxical, but presenting in groups can help the buy-in of the leader by hearing other people's responses to the information as well. In this situation, the initiative may start off with an uphill effort since so many authorities will still have expectations of the institution, but increasing awareness of the problems can multiply positive change.

e) Not interested in clinician wellbeing, profit is the focus. Try what you can do in your circle of influence to see what wins can be accomplished. Mutual support among clinicians coming up with ideas locally may be a way to start, as not much enthusiasm will be coming from senior leadership. Emphasis on financial impact of burnout may help some efforts to grow, but needed resources assigned to make broader systemic changes occur are less likely.

f) Not too interested, worried what their boss may think, not feel secure in own position, risk aversive to own career. Find opportunity to start individually based programs (like seminars) to work on wellness issues, have safe forums for clinicians to be able to bring up occupational stress and its effects. Work upon achieving the critical mass of similarly minded people needed, so that the need for addressing clinician wellbeing pops up in multiple meetings, and in multiple venues. When general awareness of the problem gets to this level, leader may po- 
litically be more willing to take risks as senior leadership is aware of the problem.

\subsection{Becoming a Change Agent for a "Bottom Up" Initiative}

Become aware of what you as an individual can achieve within your circle of influence. These actions may increase the number of people who also see the organizational blind spots that you see and have desire to do something about this with you. Avoid emotion and use science (like HFE) in making the case to leadership for change.

Keep in mind your own limitations and be careful to sustain your health. Despite knowing how devastating clinician burnout can be to your colleagues, understand your own limitations. Become aware of your stress warning signs that signal you may need to back off from this project for a while and try again later especially if resistances to change are strong. Sometimes previously made ideas will become easier to implement with time, the more people that accumulate who think similarly.

Common resistances to adopting a clinician wellness program can be disheartening (Table 2). It may be helpful to see a sample to realize you are not alone and there are approaches that can address these resistances (Table 3).

Table 2. Common resistances to adopting innovation.

- How are you going to pay for that?

- Sorry, there is no money.

- The hospital accreditation agency is coming, now's not a good time.

- Sorry too busy to meet, how about in three months?

- That's not the way things are done.

- We were expected to work hard. Back in my day we had to take call every other night.

- You need to draw up a proposal to justify the spending.

o Too long, shorten it up.

o The Budget Committee says no.

- Draw up another proposal. Make it shorter, they don't have time to read this.

o The Budget Committee says no.

Table 3. General principles of approach to resistance.

- Be balanced, not emotional. Use science.

- Two people approach leader together when possible. Helps legitimize the concern.

- All death threats/blocks to the innovation have to be addressed. Is it lack of understanding of the problem or being sabotaged because of leadership issues?

- Think how to neutralize the block respectfully and without offending.

- All naysayers/blockers have to be listened to. Hear and integrate their concerns into solutions when feasible.

- Find something to agree about. Are there unifying goals that can overcome the resistance?

- Show how the innovation would achieve or improve the usual goals of the institution.

- What are the down sides of things remaining the same?

- Use "leadership speak" where you can. Address what is most important to leaders and use the terms they use. 


\section{Foundations of Organizational Interventions}

\subsection{Overview}

There are a number of organizational paths forward but it is helpful to keep in mind your organization's readiness to change, leadership support and buy-in. An institution may use one or more of the organizational methods published that is statistically significant alone or in meta-analysis where, one method is studied at a time (See Table 1). Shanafelt and Noseworthy give an appealing high level structure and approach that lays out suggested of steps of approach [29]. This manuscript will discuss a new application of HFE concepts which gives a mechanistic framework that can be applied at multiple levels of leadership to reduce occupational stress and burnout.

For a healthcare system to be effective in the reduction of clinician burnout and improve wellness, there needs to be an organizational plus individual approach. This combined approach would include two forms of coping with stress:

1) Primary Control Coping-Fix the problems causing the severe and chronic occupational stressors;

2) Secondary Coping-Help the individual to find ways to cope with ongoing stressors [30].

\subsection{Six Major Work Stressors Causing Burnout}

Six major categories of occupational stressors caused by the institution can be understood through the work of Maslach and Leiter [31]. Understanding these factors can help design mitigating strategies to help your unique institution.

1) Excessive work load-physical, cognitive and emotional;

2) Lack of Control-lack of autonomy and ability to influence work environment;

3) Poor balance between effort and reward-material and intangible rewards;

4) Lack of community-culture of mutual appreciation and teamwork-which gets worse the busier they are;

5) Lack of fairness of resources and justice;

6) Value conflict-moral distress of having to participate in unethical or suboptimal care practices, leading to moral injury.

\subsection{Cognitive Load Theory Applied to Mechanisms of Burnout}

Brief review of Cognitive Load Theory as a human factor science can give usable language for describing the mental (cognitive) load that is mentally taxing and distinguish what mental load is essential and what is not. With excessive mental load, errors are more likely to happen. Targeting a reduction of unnecessary mental load can decrease occupational stress, help efficiency and patient safety as well as make a scientific case for why hospital patient safety efforts should be concerned about clinician burnout.

- Intrinsic cognitive load (ICL) is the inherent level of difficulty, thought to be an immutable load e.g. cognitive load needed to diagnose congestive heart 
failure.

- Germane cognitive load (GCL) includes planning, doing next steps to manage care including emotional work with patients and families.

- Extraneous cognitive load (ECL) is the cognitive load that is able to be reduced by better design. An example is the mental load imposed by the (lack of) organization of multiple disparate sources for mandatory tasks [32]. For current healthcare, an example of ECL is the multiple requirements that come from a variety of authorities that are not strategic or harmonized to achieve the intended purpose in the setting of limited resources. The amount of mental effort needed to determine what needs to be done to comply, whom to notify, how to get the time needed to accomplish it, deciding what work issues can be put off in order to accomplish the requirement is all extraneous to the tasks and uses up brain resource. Another related example is the mental effort used in the learning curve of understanding how to operate each requirement's education vendor software to successfully navigate and record compliance. The greater the ECL, the more brain power gets used and is diverted from use in safe patient care [33]-[40].

Brain executive function of the clinician is why patients come to see us. It is used to be able to diagnosis and treat as well as express compassion, and show "Aequinimitas"-being steady in the midst of patient's physical pain, blood loss, emotional pain of the patient and family. It is also used in maintaining professionalism in the midst of being treated poorly by colleagues, staff, patients or their families. Therefore a key organizational intervention is to decrease extraneous cognitive load on clinicians that depletes available executive function.

\subsection{Human Factors/Ergonomics in Healthcare Leadership and Management}

Often HFE is applied to physical work environments work flows, human-technology screen interfaces, surgical safety checklists, designing safety into medical devices etc. This paper will promote an application of HFE by healthcare leaders that is pre-clinician, i.e. taught to healthcare leaders, managers and quality and safety professionals to give them tools to create better and safer work conditions. See Figure 2 for examples of how and where hospital leaders can apply HFE to optimize the efficiency and wellbeing of HCWs. Subsequently, leader HFE education promotes wellbeing of those they lead while minimizing the risk of latent errors and burnout-inducing environment that could come from their decisions. In one institutions experience, teaching Human Factor Based Leadership (HFBL) seminar series, ninety percent of material on average was deemed to be useful for all healthcare leaders. One hundred percent $(21 / 21)$ of attendees agreed that the material included would substantially decrease clinician burnout and latent error in the organizational system [17]. Reader is referred to reference 20 for more content detail; however examples of application of HFBL are demonstrated in Table 4.

Many of the well-intended interventions to improve specific elements of quality 


\begin{tabular}{|c|c|c|c|c|}
\hline \multicolumn{5}{|c|}{$\begin{array}{l}\text { Leadership Application of Human Factors/ Ergonomics (HFE) } \\
\left.\text { Pre-Provider Factors ( } 7^{\text {th }} \mathrm{P}\right)\end{array}$} \\
\hline \multicolumn{3}{|c|}{$\begin{array}{c}\text { Pre -Provider Factors ( } 7^{\text {th }} \text { P) } \\
\text { Induced by External System and Organization }\end{array}$} & \multicolumn{2}{|c|}{$\begin{array}{l}\text { Usual Human Factors in } \\
\text { Healthcare Delivery (6 Ps) }\end{array}$} \\
\hline $\begin{array}{l}\text { National } \\
\text { State } \\
\text { Industry: } \\
\text { Regulations } \\
\text { Mandates } \\
\text { Laws } \\
\text { Policies }\end{array}$ & \multirow{3}{*}{$\begin{array}{l}\text { What can } \\
\text { leaders do } \\
\text { to avoid } \\
\text { overloading } \\
\text { the HCW? }\end{array}$} & \multirow{4}{*}{$\begin{array}{l}\text { Hospital Leadership Overview } \\
\text { - Sets institutional culture } \\
\text { - View experience of providing care as Human Factors/ } \\
\text { Ergonomics (HFE) in the delivery of care } \\
\text { - Decisions on initiative roll outs support ability to comply during } \\
\text { work time } \\
\text { - Application of participatory management to get healthcare } \\
\text { worker (HCW) input on matters that affect their daily } \\
\text { operations and work/life integration. } \\
\text { - Apply HFE for medical equipment procurement, attain more } \\
\text { intuitive devices, standardized across the institution, require } \\
\text { low cognitive demand to utilize. } \\
\text { - Budget HCW cognitive resources to be reserved for use in } \\
\text { care of the patient. } \\
\text { - Workflow designs that optimize HCW level of training and } \\
\text { minimize work that can be done by others. } \\
\text { - Policies that recognize human limitation in the context of finite } \\
\text { resources. } \\
\text { - Space, staff allocation and workflow designs that minimize } \\
\text { staff, patient and family distress and create safe work } \\
\text { environments. }\end{array}$} & $\begin{array}{l}\text { Provider } \\
\text { and other }\end{array}$ & Peripherals \\
\hline \multirow{3}{*}{$\begin{array}{l}\text { Hospital System } \\
\text { Mitigate or Amplify } \\
\text { occupational stress? }\end{array}$} & & & HCWs & Policy \\
\hline & & & $\begin{array}{l}\text { Work outside } \\
\text { of work } \\
\text { Must be minimized }\end{array}$ & Patient \\
\hline & & & $\begin{array}{l}\text { Off time } \\
\text { Must be protected: } \\
\text { Physical, emotional } \\
\text { and cognitive } \\
\text { restoration }\end{array}$ & \\
\hline
\end{tabular}

Adapted from Lowe CM. Accidents waiting to happen: The contribution of latent conditions to patient safety. Qual Saf Health Care 2006; 15 (Supple 1):i72-i75. Croskerry P, Abbass A, Wu AW. Emotional Influences in Patient Safety 2010. J. Patient Saf. 6 (4): 199-205.

Figure 2. Leadership application of Human factors/Ergonomics (HFE) helps mitigate the severe occupational stress that comes from national state and industry regulations, laws and policies. The total impact on clinicians from satisfying requirements from multiple authorities can be mitigated by how leaders organize and implement methods of compliance that recognize human strengths and limitations.

safety or value, when taken in total, paradoxically are contributing to healthcare system dysfunction, by means of cumulative effect [41]. Understanding how essential brain power is drained from processing information that is extraneous to the clinical care helps leaders apply ways to prevent this from happening in different clinical settings. Extraneous cognitive load affects next clinical decisions made, quality and safety of decisions, has a personal toll on the clinician and sustainability of practice and yet is a modifiable a risk factor for burnout and error prevention.

\section{Organizational Action Template}

Once moving forward, an organizational structural action plan is needed. A suggested action template is below.

1) Appoint a Medical Director of your wellness efforts with protected time. Best if have an Associate Medical Director with protected time as well, ideally one director from a cognitive specialty, the other from a procedural specialty, reporting structures of Medical and Associate Medical Director to Chief Medical officer (CMO) or Chief Executive Officer (CEO) of the organization.

2) Meet with key interested organizational stakeholders. It is highly recommended that patient safety and quality professionals, information technology, risk management, patient experience professionals and finance professionals are part of the audience. Senior leadership is present. Harness the power of leadership. 
Table 4. Leadership application of HFE to reduce extraneous cognitive load.

Brain Resource Used
Focusing attention
Decision making (no matter
size of decision)
Sorting, classifying
treated, maintaining calm in
setting of bleeding injury pain
Emotion Work: Dealing with
bad outcomes, distressed
patients and families
Maintaining goals and
Updating working memory
then getting back on track

FROM NASA TLX:

High mental demand

High temporal demand

\section{High frustration}

High effort

Lack of physical, cognitive and emotional restoration

Examples of Impact

- Reason for patient visit vs. Meaningful Use (MU), Screening questions.

- EMR clicks to find and achieve action. When to fit in educational mandatories.

- Lab result overload of their in-basket of EMR, from non-selectively copying in all clinicians associated with patient's care, creating diffusion of responsibility risk of oversight.

- Interruption of nurses passing meds Interruption of clinicians in emergency room.

- EMR design requires multiple page interfaces before can do what intended. Clinical data stored in non-intuitive locations.

- Patient or family member threatening clinician. Series of many patients to see with severe injury or illness.

- Death in Operating Room, next case wheeled in, giving support to grieving families.

- Chaotic workflows, busy-work conflated with virtuous work of clinical calling. Fit in teaching, research.

- Shortened patient visits, push for high Relative Value Units (RVUs).

- Hospital purchased IV pumps from multiple vendors and operate differently.

- Effort required to work around the poor designs and workflows in order to do their best to take good care of patients.

- Writing the day's clinical notes in the evening or weekends when home. Maintenance of Certification (MOC) requirements over and above what occurs in daily practice. Not able to be with significant other, children, friends or outside hobbies.

\section{Institutional Applications of HFE}

- Support staff do MU and screening questions. Patient entered data. Minimize Best Practice Alerts (BPAs) in Electronic Medical Record (EMR) which interrupt attention. Call attention to key issues but avoid demanding attention as BPAs do.

- Minimize EMR clicks. Institutional Mandatory Management system to support achievement during work time, satisfice requirements to what is needed to comply.

- Policy banner: "Clinician that orders test follows up on it". Include ordering clinician name.

- Nursing medication room, cone of silence. System of pended staff questions for clinician to be addressed between tasks and not break train of thought.

- EMR Dashboards that create action pathways accessible from one page interface. Health Information Management team collaborate with clinicians for design of labelling information and its location.

- Close collaboration with Public Safety or Security team, de-escalation training. Surrounding culture of esprit de corps supporting each other tangibly, emotionally and informationally.

- Make debriefing routine, create peer support groups, and build institutional culture of expecting clinician to be able to take a break to recuperate.

- Leadership work with clinicians to create better workflows and get credit for all missions of the institution (e.g. teaching, patient care, research).

- Organizational structure for ongoing input from rank and file clinicians to improve workflows and work/life integration.

- Standardization of IV pump equipment across the institution. Participatory management of clinician input into device purchasing.

- Human Factor Based Leadership to understand how to reduce and prevent unnecessary efforts.

- Work outside of Work (WOW) campaign to reduce WOW. Optimize EMR usability, work with Risk Management, Billing, and Compliance and Patient Safety efforts to eliminate excessive documentation. As an institution, take on complexities of MOC with framework to creatively get credit for MOC requirements from common clinical and education activities that normally occur, double-purposing actions where possible. 
3) Brief presentation on issues involved in clinician burnout-internal or external speaker. Discussion period is important to build in.

4) Work on individual based interventions like wellness seminars as a start, then plan for organizational interventions. Starting this way helps gain critical mass of more clinician and administrator change agents.

5) Determine what the major clinician concerns have been, examples. What is been tried? Any successes?

6) Start with greatest emphasis currently: Financial, efficiency, retention and turnover, patient satisfaction, lack of clinician well-being.

7) Assessment of the state of the institution

a) Anonymous survey with cover letter from leader that they are committed to action. Include:

i) Burnout assessment by any validated tool.

ii) Write in questions that ask:

1) What are the most meaningful things to you in your work?

2) What are two major stressors in your work?

3) What are two practical suggestions the institution can do, to reduce the stress and improve clinician satisfaction?

b) Means for analysis for both quantitative and qualitative analysis of themes.

c) Share aggregate information back with clinicians to demonstrate transparency, authenticity and to gain trust.

d) Consider clinician meetings that present the findings of the anonymous survey for open discussion. Take notes on further suggestions.

8) Progressive phases of interventions. Start with quick wins first, then prioritize interventions by means of impact and feasibility study from the list of suggestions. See reference [24] for more prioritization method details. Roll out of interventions over a 5 year plan based upon impact and feasibility considerations.

9) Set up organizational processes for clinician input at multiple access points throughout the medical center to submit electronic medical record (EMR) or workflow concerns for administration review and action. Implement participatory management models that allow input from clinicians on medical center, department or division decisions that affect their work flows.

10) Form a Wellness Strategic Planning Work Group (WSPWG) to analyze survey and clinician meeting input and make recommendations to senior leadership. WSPWG comprised of clinicians (attendings, residents, nurse practitioners, physician assistants, and nurses), information technology and communications personnel, and administrators. WSPWG serves as an organizational structure that will synthesize recommendations to senior leadership based upon themes and other findings of the survey. It also serves as an ongoing think tank and processing center to address hospital level work/life integration concerns.

11) Over time present projects done, possible solutions, determine if solutions scalable and transferrable to other areas. Start accumulating a file of successful projects that can inspire and guide projects in other locations. 
12) Human Factor Based Leadership seminars content is critical to help leaders understand how they can help in this high stress work environment [17]. Main points to keep in mind:

a. Brain power (neural resource, synaptic capital) is a limited resource and leaders can learn how to budget this resource like they would have to budget money, space, personnel, etc. [See earlier section for examples].

b. Patient safety, quality efforts and wellness efforts should collaborate closely which will synergize improvement in both areas. C-Suite and senior leaders have a strong role in helping to unite these initiatives.

13) Set up Department and Division Wellness Representatives program to liaison with WSPWG and Department Chair, Division Chief as well as EMR super-user or builder. The Department/Division wellness reps become a subgroup of WSPWG. This creates many opportunities for local participatory management models in departments or divisions and serves as a two-way communication conduit between WSPWG and Departments/Divisions.

14) Communications Office collaboration to keep all clinicians apprised of what is being done. When communicating to clinicians, tie in any new wellness initiative to concerns previously made known to administration: "we heard you and this is what we are doing for you" message.

15) Collaboration with existing wellness efforts sectors includes Employee Assistance Program representatives, occupational medicine. If you have a Chief Wellness Officer, they can foster close collaboration to synergize strategic plan across the medical center.

16) Circle back to the power of C-Suite and senior leadership involvement and intermittently they should be physically present at wellness meetings to show support and accelerate change.

17) Participatory management (PM) models at hospital and department/division levels.

\section{Conclusion}

Organizational interventions to improve clinician burnout in combination with individual interventions to reduce and manage burnout have enormous potential to help protect clinician wellbeing, reduce risks of error and, bring satisfaction and sustainability in taking care of patients. Human Factors/Ergonomic (HFE) science has an important role in the training of leaders and managers. It can help them successfully lead in a highly stressful and ever changing healthcare work environment. Patient care quality and experience is affected by the work environment. Organizationally, an expected outcome is being a safe and desirable place to work. This practice setting would attract and retain excellent clinicians, able to give the best care to their patients. Esprit de corps is created that moves the institution forward as an engaged team. Improved partnerships between clinicians and administrators benefit clinicians, administrators and the patients they serve. 


\section{Acknowledgements}

The author would like to acknowledge the administrators and clinicians who have worked together on improving the experience of providing care to patients, recognizing human strengths and human limitations in healthcare delivery. Thank you to Arthur S. Hengerer MD, in appreciation of his thoughtful review and suggestions for this paper.

\section{Conflicts of Interest}

The author declares no conflicts of interest regarding the publication of this paper.

\section{References}

[1] Patel, R.S., Bachu, R., et al. (2018) Factors Related to Physician Burnout and Its Consequences: A Review. Behavioral Sciences, 8, 98. https://doi.org/10.3390/bs8110098

[2] Wurm, W., Vogel, K., Holl, A., Ebner, C., Bayer, D., Mörkl, S., et al. (2016) Depression-Burnout Overlap in Physicians. PLoS ONE, 11, e0149913.

https://doi.org/10.1371/journal.pone.0149913

[3] Dyrbye, L.N., Thomas, M.R., Massie, F.S., Power, D.V., Eacker, A., Harper, W., et al. (2008) Burnout and Suicidal Ideation among U.S. Medical Students. Annals of Internal Medicine, 149, 334-341. https://doi.org/10.7326/0003-4819-149-5-200809020-00008

[4] Oreskovich, M.R., Kaups, K.L., Balch, C.M., et al. (2012) Prevalence of Alcohol Use Disorders among American Surgeons. The Archives of Surgery, 147, 168-174. https://doi.org/10.1001/archsurg.2011.1481

[5] Appels, A. and Schouten, E. (1991) Burnout as a Risk factor for Coronary Heart Disease. Behavioral Medicine, 17, 53-59. https://doi.org/10.1080/08964289.1991.9935158

[6] Centers for Disease Control and Preventions (2008) Exposure to Stress: Occupational Hazards in Hospitals. https://www.cdc.gov/niosh/docs/2008-136/default.html

[7] Salyers, M., Bonfils, K., Luther, L., Firmin, R., et al. (2017) The Relationship Between Professional Burnout and Quality and Safety in Healthcare: A Meta-Analysis. Journal of General Internal Medicine, 32, 475-482. https://doi.org/10.1007/s11606-016-3886-9

[8] Privitera, M.R., Plessow, F. and Rosenstein, A.H. (2015) Burnout as a Safety Issue: How Physician Cognitive Workload Impacts Care. National Patient Safety Foundation e-News.

http://npsf.site-ym.com/blogpost/1158873/224974/Burnout-as-a-Safety-Issue--How -Physician-Cognitive-Workload-Impacts-Care

[9] Lyndon, A. (2015) Burnout among Health Professionals and Effect on Patient Safety. AHRQ PS Net. Perspectives on Safety.

https://psnet.ahrq.gov/perspective/burnout-among-health-professionals-and-its-eff ect-patient-safety

[10] Privitera, M.R. (2018) Is Burnout a Form of Depression? It's Not That Simple. Medscape Psychiatry. Requested Commentary. https://www.medscape.com/viewarticle/896537 
[11] Privitera, M.R., Atallah, F., et al. (2018) Physicians Electronic Health Records Use at Home, Job Satisfaction, Job Stress and Burnout. Journal of Hospital Administration, 7, 52-59. https://doi.org/10.5430/jha.v7n4p52

[12] National Academies of Sciences, Engineering and Medicine (2019) Taking Action against Clinician Burnout: A Systems Approach to Professional Well-Being. The National Academies Press, Washington DC. https://doi.org/10.17226/25521

[13] Panagioti, M., Panagopoulou, E., Bower, P., et al. (2017) Controlled Interventions to Reduce Burnout in Physicians: A Systematic Review and Meta-Analysis. JAMA Internal Medicine, 177, 195-205. https://doi.org/10.1001/jamainternmed.2016.7674

[14] West, C.P., Dyrbye, L.N., et al. (2016) Interventions to Prevent and Reduce Physician Burnout: A Systematic Review and Met-Analysis. The Lancet, 388, 2272-2281. https://doi.org/10.1016/S0140-6736(16)31279-X

[15] DeChant, P.F., Acs, A., et al. (2019) Effect of Organization-Directed Workplace Interventions on Physician Burnout: A Systematic Review. Mayo Clinic Proceedings: Innovations, Quality \& Outcomes, 3, 384-408. https://doi.org/10.1016/j.mayocpiqo.2019.07.006

[16] Merlino, J. (2015) The Responsibility Matrix: A Strategy for Stronger Physician/Administrator Partnerships.

https://www.beckershospitalreview.com/hospital-physician-relationships/the-respo $\underline{\text { nsibility-matrix-a-strategy-for-stronger-physician-administrator-partnerships.html }}$

[17] Privitera, M.R. (2019) Human Factor Based Leadership: Critical Leadership Tools to Reduce Latent Error and Burnout in a Time of Accelerating Change. Health, 11, 1224-1245. https://doi.org/10.4236/health.2019.119095

[18] FAA Aviation Maintenance Technician Handbook-General. Chapter 14. Human Factors FAA Safety. https://www.faasafety.gov/files/gslac/courses/content/258/1097/AMT_Handbook_A ddendum_Human_Factors.pdf

[19] International Ergonomics Association. https://iea.cc/what-is-ergonomics/

[20] White, W.L. (1997) The Incestuous Workplace: Stress and Distress in the Organizational Family. Hazelden Information \& Educational Services, Center City Minnesota.

[21] Martin, R. (2007) How Successful Leaders Think. Harvard Business Review, 85, 60-66. https://doi.org/10.1108/sd.2007.05623jad.007

[22] von Bertalanffy, L. (1968) General System Theory: Foundations, Development, Applications. George Braziller Inc., New York.

[23] Berwick, D.M., Nolan, T.W. and Whittington, J. (2008) The Triple Aim: Care, Health, and Cost. Health Affairs, 27, 759-769.

https://doi.org/10.1377/hlthaff.27.3.759

[24] Privitera, M.R. (2018) Addressing Human Factors in Burnout and the Delivery of Healthcare: Quality \& Safety Imperative of the Quadruple Aim. Health, 10, 629-644. https://doi.org/10.4236/health.2018.105049

[25] Bodenheimer, T. and Sinsky, C.A. (2014) From Triple to Quadruple Aim: Care of the Patient Requires Care of the Provider. The Annals of Family Medicine, 12, 573-576. https://doi.org/10.1370/afm.1713

[26] Sikka, R., Morath, J.M. and Leape, L. (2015) The Quadruple Aim: Care, Health, Cost and Meaning in Work. BMJ Quality \& Safety, 24, 608-610. https://doi.org/10.1136/bmjqs-2015-004160

[27] Kohn, L.T., Corrigan, J. and Donaldson, M.S. (2000) To Err is Human: Building a 
Safer Health System. National Academy of Sciences, Washington DC. https://pubmed.ncbi.nlm.nih.gov/25077248/

[28] Montgomery, A. (2014) The Inevitability of Physician Burnout: Implications for Interventions. Burnout Research, 1, 50-56. https://doi.org/10.1016/j.burn.2014.04.002

[29] Shanafelt, T.D. and Noseworthy, J.H. (2017) Executive Leadership and Physician Well-being: Nine Organizational Strategies to Promote Engagement and Reduce Burnout. Mayo Clinic Proceedings, 92, 129-146. https://doi.org/10.1016/j.mayocp.2016.10.004

[30] Weisz, J.R., McCabe, M.A. and Dennig, M.D. (1994) Primary and Secondary Control among Children Undergoing Medical Procedures: Adjustment as a Function of Coping Style. Journal of Consulting and Clinical Psychology, 62, 324-332. https://doi.org/10.1037//0022-006X.62.2.324

[31] Maslach, C. and Leiter, M.P. (1997) The Truth about Burnout: How Organizations Cause Personal Stress and What to Do about IT. Jossey-Bass, San Francisco.

[32] Sweller, J., Ayres, P. and Kalyuga, S. (2011) Cognitive Load Theory. Springer, New York. https://doi.org/10.1007/978-1-4419-8126-4

[33] MacNamee, K. (2020) Analysis: A Neurocognitive Approach to Developing Safer Medical Devices. Biomedical Instrumentation \& Technology, 54, 28-36. https://doi.org/10.2345/0899-8205-54.1.28

[34] Privitera, M.R., Rosenstein, A.H., Plessow, F. and LoCastro, T.M. (2015) Physician Burnout and Occupational Stress: An Inconvenient Truth with Unintended Consequences. Journal of Hospital Administration, 4, 1-8. https://doi.org/10.5430/jha.v4n1p27

[35] Arnsten, A.F.T. (1998) The Biology of Being Frazzled. Science, 280, 1711-1712. https://doi.org/10.1126/science.280.5370.1711

[36] Plessow, F., Fischer, R., et al. (2011) Inflexibility Focuesed under Stress: Acute Psychosocial Stress Increases Shielding of Action Goals at the Expense of Reduced Cognitive Flexibility with increasing Time Lag to the Stressor. Journal of Cognitive Neuroscience, 23, 3218-3227. https://doi.org/10.1162/jocn_a_00024

[37] Wickens, C.D. and Hollands, J.G. (2000) Engineering Psychology and Human Performance. 3rd Edition, Prentice-Hall, Upper Saddle River, NJ.

[38] Rock, D. (2009) Your Brain at Work: Strategies for Overcoming Distraction, Regaining Focus and Working Smarter All Day Long. Harper Collins Publishers, Broadway, NY.

[39] Levitin, D.J. (2014) The Organized Mind. Thinking Straight in the Age of Information Overload. Penguin Random House LLC, New York.

[40] Hart, S.G. and Staveland, L.E. (1988) Development of NASA-TLX (Task Load Index): Results of Empirical and Theoretical Research. Advances in Psychology, 52, 139-183. https://doi.org/10.1016/S0166-4115(08)62386-9

[41] Sinsky, C.A. and Privitera, M.R. (2018) Creating a "Manageable Cockpit" for Clinicians: A Shared Responsibility. JAMA Internal Medicine, 178, 741-742.

https://doi.org/10.1001/jamainternmed.2018.0575 